\title{
Compound Formation and Hydrogen Activity at Sulfided Catalysts: A Combined Surface Science and Quantum Chemical Approach
}

\author{
J. Paul ${ }^{\mathrm{a}}$, H. Akpati ${ }^{\mathrm{b}}$, P. Nordlander ${ }^{\mathrm{b}}$, W.S. Oh${ }^{\mathrm{c}}$, D.W. Goodman ${ }^{\mathrm{c}}$ and B. Demirel ${ }^{\mathrm{d} s}$ \\ ailkent University, Chemistry, Bilkent 06533, Ankara, Turkey

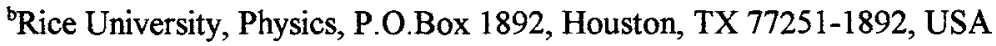 \\ 'Texas A\&M University, Chemistry, College Station, TX 77843-3255, USA \\ 'University of Utah, Chemical and Fuels Eng., Salt Lake City, UT 84112, USA \\ ${ }^{8}$ for correspondence, present address: \\ Center for Applied Energy Research, 3572 Iron Works Pike, Lexington, KY 40511-8433, USA
}

\begin{abstract}
This paper discusses the hydrogen activity of sulfided metal catalysts supported on modified carriers. Surface characterization and theoretical modeling are combined with published studies of planar models to deduce a conceptual model for hydrogen dissociation and mobility on industrial hydrogenation catalysts. The hydrogen activity of metalsulfides is compared with that of the corresponding transition metals.
\end{abstract}

\section{TEXT}

Sulfided catalysts are workhorses of the refining industry. They are, among other processes, used for hydrogenation reactions at elevated temperatures. This activity as well as the activity for hydrodesulfurization (HDS) and hydrodenitrogenation (HDN) is intimately related to the hydrogen activity of metal sulfides i.e. to the properties of adsorbed atomic hydrogen and the rate of hydrogen dissociation at the surfaces of these compounds [1]. The present work is an attempt to model these properties by advanced electron structure methods, experimental and theoretical. The work is part of a project for the production of gasoline components from polyaromatic hydrocarbons (PAH) [2]. Other parts address conversion rates and turnover frequencies, mass balance and surface intermediates [3].

A sulfided catalyst consists of three dimensional metal sulfide particles bound to a supporting oxide. Sulfidation lowers the dispersion of an impregnated metal phase by the strong 
driving force for sulfide formation and structural methods reveal particulates of stoichiometric compounds. This coalescence opens areas of the supporting oxide for adsorption, an important secondary result with relevance for cracking reactions at the modified sites created by electronic modifications in titania-alumina or silica-alumina catalysts. These modified sites also act as dispersion agents for the metal in its oxidic state, but this property has less significance after sulfidation than after mild reduction, sulfidation provides an extra incentive for coalescence.

Modified or acidic sites are centra for dehydrogenation reactions, a property commonly used to produce unsaturated compounds from alkanes [4]. Hydrogenation of 1-methylnaphthalene was found to proceed effectively over a sulfided NiMo catalyst supported on a titanium doped alumina, but only remote quantities of cracking products were observed [2]. This indicates that the acid sites were blocked either by supported metals or carbonaceous species. This motivates our interest in isolated species on models of the carrier.

The present work includes experimental characterization of the effects of sulfidation by static weight changes for stoichiometry and by photoemission spectroscopy (ESCA) for chemical characterization. Hydrogen adsorption is modeled by calculations.

Transition metals have superior activity for hydrogenation reactions below the temperature for recombinative desorption of hydrogen. Above this temperature these materials instead possess dehydrogenation activity, an often activated process, due to weakening and eventual dissociation of $\mathrm{C}-\mathrm{H}$ bonds from overlap with the surface electron density. Unsupported metals are less useful for high temperature hydrogenation reactions due to the low surface coverage of atomic hydrogen, a result of molecular desorption after $\mathrm{H}-\mathrm{H}$ recombination, not because of an altered ability for hydrogen dissociation. Hydrogen bond-weakening and dissociation is a result of electronic overlap, a property only marginally affected by temperature. Hydrogen dissociation occurs because hydrogen orbitals overlap or hybridize with d-orbitals of appropriate symmetry when the molecule approches the surface with the $\mathrm{H}-\mathrm{H}$ axis parallel to the surface. Mixing between the metal d-states and hydrogen $\sigma$ and $\sigma^{*}$ orbitals bring the latter partly below the Fermi level. This results in bondweakening and eventual dissociation.

Atomic hydrogen from the above process is trapped in the surface region. The tail of electron density gives at a certain height above the surface a most favorable electron density, well formulated in the effective medium approach [5]. Further penetration into the metal is obstructed by repulsion from the higher density. Motion away from the surface is also hindered, atomic desorption is only observed at drastically higher temperatures, a well known phenomenon for the production of atomic hydrogen in diamond film synthesis. Atomic hydrogen is, as a consequence, trapped in the surface region. The adsorption potential for atomic hydrogen is predominantly a result of the free or sp-electron density with a smaller perturbation from d-states. The sp-density is evenly spread in the surface plane with only a small corrugation due to the atomic cores. This means that no major barriers exist for diffusion along the surface, resulting in a comparatively low temperature for recombinative desorption.

The maintained high dissociation rate at elevated temperatures means that atomic hydrogen can become available as long as a competitive process is more effective than 
recombinative desorption. This is the concept of hydrogen spillover. Hydrogen spillover is promoted by small particle sizes and enhanced temperatures. The small particle size means an enhanced chance for hydrogen diffusion across the boundary between the metal particle and the supporting oxide. Large particles and poor dispersion instead promote hydrogen recombination. An enhanced temperature is necessary to boost the diffusion rate across the metal/support barrier and further via the the support material.

The key feature of metal sulfides is to maintain a high coverage of atomic hydrogen at the elevated temperatures of hydrotreatment reactors. This means that recombinative desorption is quenched but not necessarily that the rate of hydrogen dissociation on the terminated metal sulfide particles is high. Different concepts for hydrogen dissociation on sulfided catalysts exist. One being the existence of special sites directly on the metal sulfide particles, these sites are often envisioned as more metallic and thus more active for hydrogen dissociation than the bulk sulfide. Another concept is a dual function catalyst where a supported well dispersed metal provides atomic hydrogen, followed by hydrogen diffusion over to a second metal, in its sulfide form, where the hydrogenation occurs [6]. Either model is possible. Typical catalysts are bimetallic with one metal, Mo or $\mathrm{W}$, forming the dominant sulfide and another metal, $\mathrm{Co}$ or $\mathrm{Ni}$, acting as wetting agent between the metalsulfide and the support or possibly forming a separate metallic phase with high capacity for hydrogen dissociation. The hydrogenation rate usually peaks at a Mo:Co or W:Ni ratio of $3: 1$ [1]. The reducing environment of hydrogenation reactions can easily reduce any metal still in the oxidic state as well as create special sites at the surface of $\mathrm{MoS}_{2}$ or $\mathrm{WS}_{2}$ particles. The problem with each model is that they are often deduced from kinetics data and it is non-trivial to know which barrier a certain apparent energy term comes from.

The present calculations center around cluster models of hydrogen adsorption on metal sulfide particles. The electron structure is solved in a local density approach with numerical radial parts of the atomic wavefunctions and a new dense and thus accurate integration grid. We consider surface coverages as well as penetration into the bulk with reference to the known 3D structure of the sulfide particles and the role this morphology plays for the hydrogen activity. We also refer to previous studies of hydrogen adsorption on unsupported metals.

The structure of naphthalene and 1-Me-naphthalene, model compounds for heavy feeds, are modelled in a less computer intensive $a b$ initio code [7].

The experimental part contains weight measurements to obtain the stoichiometry of sulfides at the surface and photoemission spectra to characterize these phases. The catalysts were either retrieved from intermittent steps in autoclave studies with actual feeds or prepared in parallel batches for reactor studies and surface characterization. Quantitative measurements of mass changes during catalyst pretreatments, dehydration and sulfidation, are also prerequisites for enhanced accuracy in mass balance calculations including carbonaceous residues [3]. 


\section{REFERENCES}

1. O. Weisser and S. Landa, Sulphide Catalysts, Their Properties and Applications, (Pergamon Press, Oxford, 1973); R. Prins, V. H. J. De Beer and G. Somorjai, Catal.Rev.Sci.Eng. 31 (1989) 1; P. Grange, Catal.Rev.Sci.Eng. 21 (1980) 135; J. J. F. Scholten, A. P. Pijpers, and A. M. L. Hustings A.M.L., Catal.Rev.Sci.Eng. 27 (1985) 151; J. A. Rodriguez, S. Y. Li, J. Hrbek, H. H. Huang and G. Q. Xu, J.Phys.Chem. (in press); R. I. Declerek-Grimee, P. Canesson, R. M. Friedman and J. J. Fripiat, J.Phys.Chem. 82 (1978) 889

2. B. Demirel, PhD Thesis, Univ. of Utah (1996)

3. B. Demirel and W. H. Wiser, manuscript; B. Demirel and J. Paul, manuscript; S. Sayan, B. Demirel, W.H. Wiser, S. Süzer, and J. Paul, manuscript

4. G. B. McVicker, G. M. Kramer and J. J. Ziemiak, J.Catal.83(1983)286; A. Corma and B. W. Wojciechowski, Catal.Rev.Sci.Eng. 279 (1985) 29

5. P. Nordlander, S. Holloway and J. K. Nørskov, Surface Sci. 136 (1984) 59

6. A. M. Stumbo, P. Grange and B. Delmon, in Studies in Surface Science and Catalysis (Proceedings 11th ICC) 101 (1996) 97

7. B. Demirel and J. Paul, Z.Phys.Chem., submitted 